\title{
Lives rendered invisible: Bearing witness to human suffering
}

\author{
Mladjo Ivanovic ${ }^{1}$
}

Department of Philosophy, Michigan State University, mivanovi@msu.edu

DOI: http://dx.doi.org/10.5324/eip.v10i2.1919

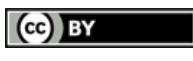

This is an open access article distributed under the terms of the Creative Commons Attribution 4.0 International License, which permits unrestricted use, distribution, and reproduction in any medium, provided the original author and source are credited.

This paper explores the ethical challenges involved in the ways public representation structures our experiences of atrocities and facilitates an adequate awareness of and response towards the suffering of others. It points out that such an analysis should not exhaust itself in answering what makes public representations of human suffering ethically suspicious and intolerable, but should rather extend this task by clarifying how the public forms sentiments about their social and political reality by elucidating under which conditions public representation promotes broader political agendas. One of the central tenets of human rights advocacy is the widespread conviction that exposure to images and stories of human rights abuse has a mobilizing effect on western audience(s) whose exposure to such knowledge can motivate them to intervene and prevent future atrocities. In order to assess the basic implications of such a conviction we must answer at least three principal clusters of questions. First, how do public representations of atrocities affect individuals and their capacities to conceive and respond to social injustices and the suffering of others? Under what circumstances may agents respond effectively to shocking content? Second, how do social powers operate within the field of perception in order to control how the viewing public is affected? And how do these effects inform and galvanize political support or opposition regarding concrete historical events? Finally, what can be said about the responsibilities of visual representation? Whose agency is it that images inform, and what reforms are necessary to make representations of suffering ethically effective means to encourage better acknowledgment of individual and collective responsibilities that would motivate the public to meet its moral and political obligations? This paper ultimately suggests that in order for politically implicated images to have an immediate critical effect on individuals and their agency, they need to cultivate alternative modes of perception.

Keywords: ethics of representation, images of atrocities, social responsibility, political obligations, perception

\section{Introduction}

In the aftermath of the atrocities of the Second World War, Adorno warned that aestheticizing human suffering reduces it to a state of banality. Referring to what he 
calls the "barbarism" of the post-Holocaust culture, Adorno doubts whether our familiar discursive practices can serve as adequate mediums to represent and understand these horrors:

The so-called artistic rendering of the naked physical pain of those who were beaten down with rifle butts contains, however distantly, the possibility that pleasure can be squeezed from it. The morality that forbids art to forget this for a second slides off into the abyss of its opposite. The aesthetic stylist principle, and even the chorus's solemn prayer, make the unthinkable appear to have had some meaning; it becomes transfigured, something of its horror removed. By this alone an injustice is done the victims (Adorno 2003: 252).

Although Adorno's characterization has been criticized by some as excessive, his statement reveals an important aspect of representing atrocity and its ties to moral evaluation. To fail to acknowledge how interpretative and symbolic representations of atrocities may undermine the tragic depth of human brutality is to overlook the complexities of the human condition, which render guilt and public witnessing into its opposite, namely, indifferent spectatorship and apathy. Implicit in Adorno's critique of the depoliticization of the Holocaust and subsequent aestheticization of violence is perhaps a reminder of a much deeper problem that plagues the relationship between aesthetics, politics and ethics: How do we make sense of the cruelty captured in images of tormented human beings? What are the moral obligations aroused through exposure to visual representation of atrocities? How do stories of human suffering mobilize our emotional capacity to understand, to make us grieve, or even to forget and remain indifferent? Perhaps Adorno, who was eager to unmask this difficulty of subjects to reconcile their experiences with events that have eclipsed their conceptual grasp, bespeaks to an inherent inability of public representation to retrace the contours of atrocities in the form of a coherent discourse.

However, Adorno's initial realization of the difficulties tied to the entanglement between aesthetics and politics remains a bit ambiguous. It seems to rest on two distinct, though related, assumptions. First, it suggests that there are various moral issues involved in the nature of knowledge/information that the visual depiction and documentation of human suffering conveys. There seems to be a dark dialectic within these instances of horror that are contextualized in visual representation, and despite the fact that photography's broad resonance has a capacity to succeed in arousing public moral outrage and provoke questions of responsibility, there also lies a danger of effectuating what it seeks to resist, namely the normalization of atrocity. Second, although representations of suffering seem to have an instrumental value in capturing public attention by accommodating viewers' imagination and emotion, such images nonetheless tell us little about the nature of the political deliberations that surround them. They don't reveal how their effects on viewers are being channeled and transformed into a specific political consciousness. If we take into consideration that exhibiting such images reduces complex geopolitical conditions to shocking but simplistic visual frames, questions arise concerning the political nature of such processes and which instances of social powers create conditions of perception that constitute subjects' comprehension of the content that these images deliver. As a result of this dilemma, the ways in which public representation affects and shapes the political and moral agency of individuals calls 
for a closer examination of the patterns, problems and inconsistencies associated with the use of images of human suffering. It is commonplace to claim that people make sense of events in the world through the information that images convey; however, in order to foster an active engagement with reality there is a need to better chart the processes through which certain depictions emerge as critical landmarks of public knowledge and imagination.

In what follows, I take this troubling relation between the invasive representation of human trauma and the political nature of the public use of such images as a convenient starting point for the theme I wish to discuss in this paper, namely, the ethical challenges involved in the ways public representation-through photography or other media-structures our experiences of atrocities and facilitates an adequate awareness and response (or a lack thereof) towards the suffering of others. In order to understand exactly what is at stake here, I point out that such an analysis should not exhaust itself in answering what makes public representations of human suffering ethically suspicious and intolerable, but should rather extend this task by clarifying how the public forms sentiments about their social and political reality by elucidating under which conditions public representation promotes broader political agendas. One of the central tenets of human rights advocacy is the widespread conviction that exposure to images and stories of human rights abuse has a mobilizing effect on western audience(s) whose exposure to such knowledge can motivate them to intervene and prevent future atrocities. In some sense, this belief for the most part also defines the relationship between aesthetics and politics. In order to assess the basic implications of such a conviction we must answer at least three principal clusters of questions. First, how do public representations of atrocities affect individuals and their capacities to conceive and respond to social injustices and the suffering of others? Under which circumstances may agents respond effectively to shocking content? What are the limits of depicting violence and human trauma? There are various issues involved in the visual depiction and documentation of atrocities and suffering. Although images can be powerful mediums that force attention and involvement, their resonance ultimately depends on where they are coming from, who wants to see them, and political contexts that support their interpretation. Perceiving a photograph that depicts a certain traumatic moment thus does not only imply an active engagement with the ways one represents a tragic event, but also implicates the viewer in the helplessness and vulnerability of others. Thus, such a perception may aestheticize the suffering of others and thereby surrender the image as spectacle, or it can politicize such representations by thinning out the experience of the spectators that may result in co-optable and sterile populist agendas and selectivism. Second, although the power of the image is built on emotional response, the specific emotions an image invokes are not predictable in themselves. They depend on topologies of social powers and the interests those powers aim to advance. Hence, what essentially undergird these relations are political processes that dictate malformed public responsiveness to instances of human suffering. Such distortions are evident, for example, in western selectivism, which handpicks certain cases of suffering to represent and address. The question we must ultimately confront, then, concerns the role of society in facilitating a space wherein moral dilemmas healthily correlate to the witnessing of injustice and atrocities. How exactly do social powers organize the field of public perception? And how does this galvanize political support or opposition to concrete 
historical events? Finally, what can be said about the responsibilities of visual representation? Whose agency is it that images inform, and what reforms are necessary to make representations of suffering ethically effective means to encourage better acknowledgment of individual and collective responsibilities that would motivate the public to meet its moral and political obligations? For this paper ultimately suggests that, in order for politically implicated images to have an immediate critical effect on individuals and their agency, they need to cultivate alternative modes of perception. Such modes, then, would challenge persisting cognitive and perceptual norms that sustain the lethargic consciousness of an audience and help induce new modes of subjectivity.

\section{Images and the contemporary hollowness of bearing witness}

How do images of human suffering affect us? How do they mobilize our attention, and elicit empathy, horror, enjoyment or anger? In spite of their moral straightforwardness, these questions are not easy to answer. Given that representations of atrocities have not only epistemological, but also ethical and political dimensions, the first step in answering these questions is to analyze how specific cultural, economic, and political conditions facilitate images as vehicles for collective knowledge and memory. Taking into account that these different aspects intersect and form the contexts in which public representation defines the standards of our social experiences, it is important to indicate the ways in which the relation between images, public knowledge, and individual agency (either through direct action, omission, or apathetic spectatorship) is constituted. In Regarding the Pain of Others, now a classic work on the uses and meanings of images of atrocities, Susan Sontag illuminates this complex relation between representations of traumatic events and an audience whose perception of violence or trauma is defined by exposure to such images. "One can feel obliged to look at photographs that record great cruelties and crimes," she writes, "to think about what it means to look at them, about the capacity actually to assimilate what they show. Not all reactions to these pictures are under the supervision of reason and conscience. Most depictions of tormented, mutilated bodies do arouse a prurient interest" (Sontag 2003: 95). Despite recent calls for a reconsideration of the validity of images of suffering as tools for socially and politically engaged photojournalism and art, there are many arguments that express doubts about the moral and political sentiments that gruesome or aesthetically appealing images of trauma may stimulate through public exhibits. On the one hand, photography can facilitate the articulation of a certain degree of moral indignation, responsibility, empathy, and compassion, forced by the fact that viewers need to decide where they stand in regard to the content that they see. On the other hand, it can generate more deleterious responses-voyeurism, shame, guilt, complicity, and indifference. The gravitation of their content towards the subject's imagination complicates both sets of emotions, for one of the most typical responses to images is an envisioned restriction of political possibilities, which needs to be contested critically.

When we consider the ordinary ways in which we think about our capacity to bear witness and understand the complexities of human conditions that result from states of vulnerability and exclusion, it soon becomes evident that how we conceive social reality and human deprivation is configured through the interpretative and 
symbolic dimensions of visual representations and the discourses that articulate them. As Barbie Zelizer has aptly observed, "[t]he compelling weight of the photograph, then, is determined by a linkage between its material and discursive dimensions, and the power created by that linkage draws us to a photo's many meanings"(Zelizer 1998: 8). This alone suggests that the multifaceted dimension of images not only helps to stabilize and support knowledge about specific events, but also fosters an alternative engagement with reality by guiding public imagination beyond the contours of mere witnessing. The use of images for political purposes relies on the assumption that complicated events can become visible and understandable through certain kinds of depiction. The graphic content of an image thus acts as a trigger for emotional release, regardless of which emotions these may be. And yet, most discourse regarding the informative role of images merits the assumption that seeing photos with gruesome content would be enough to promote action or responsiveness of some kind. The problem with this assumption is not only that it presupposes reactions in audience that are not obvious, but also that such a reduction of the political role, capacity and effect of an image to naïve spectatorship is an ideological distortion of a complex process of how images work in today's public sphere and the ways in which they affect subjects.

It is for these reasons that viewing photos of human suffering is both active and intervening, though these two aspects do not necessarily presuppose or lead to collective public action. As Jacques Rancière argues, "there is no straightforward road from the fact of looking at a spectacle to the fact of understanding the state of the world; no direct road from intellectual awareness to political action" (Rancière 2009: 75). He associates the questionable political value of photos with the lethargic consciousness of the viewing public in order to disclose the problematic complacency of the postmodern subject in his long-overdue awakening of knowledge and compassion towards the suffering of others. As a result, Rancière suggests that although gruesome images are certainly hard to bear, there is no reason why their exhibition would make an audience eager to fight against powers that cause such harrowing conditions. "The stock reaction to such images," he writes, "is to close one's eyes or avert one's gaze" (Rancière 2009: 85). Such a paradoxical situation, which defines the link between the intolerable content of an image to an awareness of the reality it expresses, is evidence of the need to challenge theoretical and political presuppositions that ground the public criticism that these images want to inform. Spectators share specific epistemic positions and they experience the content of images through their rootedness in contingent cultural, economic and political conditions that mold cognitive presuppositions that condition their perceptions of their social environments and the lives of others. Thus, Rancière's disturbing analysis of the political value of images of human suffering cannot be posed independently of the question of the political nature of the subject's comprehension and formation of his or her identity. Exposure to public representation of harrowing images is thus never a passive encounter with concrete historical events. Rather, visual content that is transmitted through images falls down on the backdrop of previous shared knowledge-both cultural and political. While such an encounter overlaps to a certain degree with previous experiences of similar events, and may even invoke sentimentality towards victims, the audience nonetheless preserves its own distinct perspective and interpretative framework, to which one has to pay attention if we are to remain critical about 
transmitted knowledge and how it may mobilize (or fail to mobilize) public attention/intervention.

As we live in a time in which acts of witnessing risk becoming distorted by various social forces that facilitate uncritical understandings of and responses to tragic events, we find ourselves challenged to apprehend the weight of knowledge that is facilitated through screened material. At the same time, displayed images offer a representational frame of human brutality and facilitate exposure and engagement more then understanding. Their reliance on effect and emotion become powerful impulses for judging such events in the public sphere. However, they are instrumentalized as effective ways to reproduce reality and shape popular imagination of atrocities, which not only enhances an acknowledgment of public responsibility regarding instances of human suffering, but also triggers "cognitive resistances" that may obscure or even make incomprehensible events that demand public attention. The capacity to presume, infer, and accept or reject meanings of images depends also on other, more symbolic forces that play an active role in diminishing individual capacity to become ethically responsive to the suffering of others. Racism, sexism, religious conservatism, national or cultural identities, and class-based stereotypes make witnessing a selective process, one that reduces complex events to a manageable size or allows us to employ our references to present-day political agendas. Referring to this selectivism, James Dawes suggests that exposure to systematic injustices and violence "triggers so many cognitive resistances, because its disorganization makes it so difficult to pack it into the standard narrative forms we use parceling knowledge of our worlds, we can be inclined to retreat into easy, familiar methods of regulative experience. For many, racism [or any other aspect of our habituation] can therefore begin to function as an almost indispensable enabling device, a useful and well-practiced response for psychically organizing the unorganizable" (Dawes 2007: 35). Such a radical contingency of reception, then, not only challenges how public representations of distant suffering can contribute to an acknowledgment of individual and collective responsibilities when confronted with human rights violations, but raises crucial questions about other possible responses to viewing and reading about violence and human suffering in general.

Despite continual references to atrocities in the media, there is a range of reasons for why such representations may no longer compel responsibility or move individuals and collectives to intervene. For instance, it is possible that, rather than motivating an audience to action, media coverage shocks and desensitizes it into a dazed passivity and denial of responsibility. As Stanley Cohen writes in his classic work on this theme:

Witnessing and reproducing the truth are cognitive projects: how to convey a reality that cannot be denied. But what if continued exposure to this reality eventually deadens our moral and emotional receptivity to further images of suffering? The populist psychology thesis of "compassion fatigue" is built upon three overlapping concepts: information overload, normalization, desensitization (Cohen 2001: 187).

In other words, although we find ourselves relying increasingly on the media and its visual depictions to help us make sense of atrocities, with time the barrage of horrifying photos loses its resonance. This deterioration takes place not only 
because such images may overwhelm the audience with the magnitude of suffering, but also because constant exposure to human deprivation and an ongoing politicization of tragic historical events (e.g. the Holocaust) normalizes them, and numbs the western audience to recurring instances of human suffering. What "compassion fatigue" teaches us, then, is that indifference to the pain of others is not so incomprehensible after all; it relies on multifaceted political performances in which cultural predilection, together with public representation, lead towards the humanization or dehumanization of the Other. As public knowledge becomes increasingly dependent on technology and political mediation, the power of images to effectively generate public awareness and support of agonies of distant others is underscored by their capacity to end in the opposite: cultural denial and further hindrance of recognition and redress. Thus, rather than inducing compassion and sympathy, they may result in resentment and indifference. Even worse, as images of atrocities become more complex and "multimediated," their open-ended interpretative and symbolic dimensions may turn audiences into voyeurs of objectified human suffering in an almost pornographic way.

This brings us back to Rancière's question of the nature of the relationship between visual representation and the effects this has on the formation of public opinion. How does such exposure shape a subject's capacity to comprehend his or her environment and the environment of others? "Why identify gaze and passivity," he writes, "unless on the presupposition that to view means to take pleasure in images and appearances while ignoring the truth behind the image and the reality outside the theatre" (Rancière 2009: 12). Interrogating the efforts of critical art to emancipate the spectator questions the attempt to traverse the abyss that divides activity from passivity by asking "if it is not precisely desire to abolish the distance that creates it" (Rancière 2009: 12). Taking into account that the mass media has an uncontested monopoly on the dissemination of information, the dependency of subjects' knowledge on broadcasting, which necessitates a constant referral to technological mediation in the formation of experience and meaning, may cause not only a complete denial of recognition towards culturally distant values, but also lead further to the preservation of cultural and political stereotypes that often have fatal consequences for the acknowledgment of our own humanitarian/political obligations towards distant people in need. As these theorists remind us, "we have only got better more subtly in looking the other way." This ignorance is not a natural process; it instead depends on the linkage between individual moral choices and the political powers that form and regulate them. A better understanding of these complex connections will help not only to uncover the network of intentions that guides injustices and clarify the unevenly shared responsibilities of those who cause these events (as well as those who witness or benefit from them), but also to collapse the distance between "us" and "them" and acknowledge how precarious human life is.

\section{Social Ontologies and Politics of Perception}

The previous pages suggest that there is no simple relationship between continual references to atrocities and public responses to the knowledge that such references convey. Despite the various ways in which representations of violence and other human rights abuses can affect an audience, the capacity to presume, accept or 
reject meaning from images is built into the image-making enterprise. Such ability to craft meaning regardless of an image's clear relevance to what is being depicted deserves further attention. Contrary to the popular assumption that "images are worth a thousand words," they actually tell us very little. Sometimes they exclude information that would otherwise be important in order to have adequate knowledge about a specific event. Thus, by leaving out cues of causality, impact, and historical contexts, and without a clear distinction between intention, neglect, accident or design that guides these events, images offer an atrophied depiction of reality. Their provocative and shocking content invites public engagement with human suffering without actually insisting that people comprehend tragedy on its own terms. In obscuring its complex historical, cultural and political aspects, public representation of atrocities usually offers a blended visual category of human-made and natural catastrophes that prevents reasoned deliberation and denies insight into the complex reality of injustice and tragedies. In this regard, although images of suffering mobilize an emotional landscape within which public reaction can take shape, this landscape is neither random nor spontaneous. One has to be sensitive to how and what kind of context is woven around the image, because it is exactly this context that gives an image its meaning and dictates its reception. In other words, the complex relations that surround a given moment captured through the camera lens-whether social, cultural, legal, moral or political in nature-determine what the image is worth, as well as the nature of public exposure and engagement. Resolving how to display specific events and determining what kind of information is needed to recognize them in a certain way is in the interest of social forces that want to advance and protect specific agendas.

We have all grown accustomed to familiar representations of atrocities and we seem to ignore the fact that an image's contingent nature can be utilized by institutional powers by manipulating the cognitive and emotional dispositions of the viewing public. As Susan Sontag argues,

(P)hotographs that everyone recognizes are now a constituent part of what society chooses to think about, or declares that it has chosen to think about. It calls these ideas 'memories' and that is, over the long run, a fiction. Strictly speaking, there is no such thing as a collective memory-part of the same family of spurious notions as collective guilt. But there is collective instruction (Sontag 2003: 85).

This gives an image a strategic use value, and renders it an intrinsic component of what Judith Butler terms a "frame" or "field of perception." Used equally to generate compassion and indifference, to accuse, distance, criticize, empathize, ridicule, manipulate, obscure, deny, neutralize, and dissociate, images thus do capture public involvement, but in strategic, and often deceiving ways. The public's capacity to make meaning out of these images therefore hinges on a nurturing of the imagination on the basis of social and political norms. To the extent that every society is defined by values and institutions that presuppose a capacity that enables social actors to influence the decisions of others, it is important to keep in mind the ways in which media outlets set up the contexts in which the interpretative and symbolic dimensions of an image form and guide individual knowledge and action. Drawing on both Foucault and Sontag, Butler's account in "Torture and the ethics of photography", and later in her book "Frames of War," expands the discourse on 
images by analyzing the ways in which atrocities and human suffering are presented to us, and more generally, how such presentations and public responses are effects of social processes of subjection that operate through visual and discursive fields. As she puts it:

Prior to the events and actions that are represented... there is an active, if unmarked, delimitation of the field itself, and so a set of contents and perspectives that are not shown, never shown, impermissible to show. They constitute the nonthematized background of what is represented, one that can only be approached through thematizing the delimitating function itself, one that allows for an exposure of the forcible dramaturgy of the state and the collaboration with it by those who deliver the visual news of the war through complying with permissible perspectives. That delimitation is part of an operation of power that does not appear as a figure of oppression (Butler 2007: 953).

Butler's claim brilliantly captures the ways in which social forces constitute human agency and enable specific regimes of intelligibility. Being a spectator/bearing witness, then, becomes a complex process wherein exposure to visual representation invokes not only an aspect of reality but positions such representation into a broader interpretative framework that actively molds the context of photography through social and political norms and practices. Since images are experienced as ways of giving meaning to our social environment, the ethical resonance of such processes depends on interpretative cultural and political codes of reference, which makes questionable the separation of representation from ongoing political agendas. Indeed, photographs serve as evidence of atrocities, but such pieces of evidence "are themselves a species of rhetoric. They reiterate. They simplify. They agitate. They create illusion of consensus" (Sontag 2003: 6). One can assume, then, that there lies a constant danger that knowledge mediated through images becomes a vehicle of ideology under the guise of representation, rather than that which references events that deserve genuine public concern and immediate action.

In order to fully grasp the weight of Sontag's and Butler's insights, one has to disclose how social ontologies work, that is, when and how one can claim a certain identity, and under which circumstances one can be visible as a human being worthy of moral concern. In the first instance, social ontology seems to be constituted within certain fields of intelligibility, fields that bring us back to questions about power, and how relations of power both sustain the subject's identity and subordinate her at the same time. Thus, as Butler argues, "there's always question prior to ontology, which is the question of power and what it orchestrates: who or what can be, has the power to be, and what allows someone or something to emerge into the field of ontology?" (Butler 2011: 86). In other words, we must ask, which conditions allow someone or something to emerge and become socially visible; which operations of these social powers decide in advance who will and who will not become a subject? If we start with the assumption that the individual is dependent on social recognition, and that his or her identity is constituted through cultural and political forces along with and in opposition to others, the failure to be recognized as a subject worthy of social concern, then, is not just a failure of civil society to successfully mediate between individuals and political powers. More fundamentally, it is the impossibility of separating the intelligibility of 
social actors from the social forces that both individualize and totalize a particular presentation of oneself. Thus, in terms of the ability of a social body to provide content for the realization of humanization (and dehumanization), disciplinary discourses alongside regimes of perception signify the totality of meanings that make any differentiation between social role and personal autonomy impossible. Such an increasing control of society over individuals results in diverse modes of behavior that are accompanied by the reduction of a subject's capacity to withstand specific cognitive and material dispositions which in turn create and sustain our response towards the suffering of others. ${ }^{2}$

Butler's gestures towards the comprehension of public imagery and politics of perception are helpful to reflect on the ways in which visual documentation of geopolitical events renders certain human beings invisible. As I suggested earlier, organization of the sensory landscape, that is, organization of how subjects perceive their environment, is not independent from the social relations that form and shape their sensory/perceptive/cognitive capacities. The invisibility of others, then, is not only constituted through the exclusion of certain social sectors that takes the form of widespread instances of disrespect (racial minorities, women, the LGBTQ community, immigrants, ethnic and religious minorities, etc.). Rather, their gradual dehumanization also depends on sensory inequality, where both aspects of social experience share the same ideological foundations of a single political process. Thus, in order to even initiate discourse on the political effects of visual imagery, one must disclose how a subject's perception is formed and sustained by social forces and the relations that they create. This is necessary because a subject's cognitive and perceptive capacities determine when and how interpretations of visual content take place and which effects it will have on a subject's agency and deliberation. This way of understanding how experience is constituted and sustained brings us back to what motivates Butler's account of ideological framing. Namely, many social and political mechanisms draw their validity and persuasiveness from symbolic cultural forces that are inherent in social upbringing, which in return strike agents as credible and usually beyond critical reasoning. Through a complex setup of representations (media, the Internet, public campaigns) the embodiment of these forces in public broadcasts are references not just to social constellations and events that unfold on a political landscape, but also to a situated practice of opportunities, performance and control. Although it may at first seem rather unattainable, the reality which comes into being through news agencies and other forms of public media transcends questions of mediation and transmission, and takes an active role in shaping public knowledge and what subjects can conceive as social reality. Thus, it remains a constant danger that those subjects whose behavior media exposure aims to affect remain vulnerable to the forces of predominant political and cultural narratives that cognitively and emotionally structure individuals according to particular, institutionally driven social contexts.

One way to grasp this process is to ask the simple question of what makes some images work better than others, and what happens with public sentiments towards those lives that are rendered invisible. The main challenge here consists in analyzing the ways in which such invisibility results from cognitive, emotional and moral reductions facilitated through complex mechanisms of visual and discursive framing. ${ }^{3}$ We might, along these lines, think that within the frame, the context of social relations postulates the possibility of political agents to be present, 
represented and representable or in turn completely denied any social intelligibility and recognition. Thus, the symbolic and structural dimensions of power condition subjects who are socially visible and recognized as individuals with intrinsic worth and meaning from those who are not. There are a variety of ways to account for such an emergence of the "subject" from the matrix of social institutions, and such processes raise important questions about individual agency, moral responsibility and the structures of institutional intelligibility. Who appears within the frame? Who is recognized and what constitutes a subject who can be recognized in the first place? In an attempt to address this problem, Butler makes a considerable effort to display how such processes of social framing orchestrate subject formation. As she puts it,

If recognition characterizes an act or a practice or even a scene between subjects, then 'recognizability' characterizes the more general conditions that prepare or shape a subject for recognition-the general terms, conventions, and norms 'act' in their own way, crafting a living being into a recognizable subject, though not without errancy or, indeed, unanticipated results. These categories, conventions, and norms that prepare or establish a subject for recognition [...] precede and make possible the act of recognition itself. In this sense, recognizability precedes recognition (Butler 2010: 5).

A close reading of these lines shows us how political forces, cultural standards and habits, forms of knowledge, procedures, spatial organizations and systems of monitoring have become the means for governing the unwanted. These categories and norms in collusion with media and other forms of representation become a field of negotiation that orchestrates the possible forms a subject may take. By introducing the notion of "frame," a notion that refers to intertwining epistemological and ontological horizons within which subjects come to be at all, Butler provides a fascinating testimony of the effects of political powers upon which a certain understanding of humanity projects itself. Such an ontological predisposition of epistemological intelligibility transforms the social space into a performative one wherein the intersection of visual control and an ethical preselection creates the conditions for the inclusion and exclusion of human beings.

\section{The Public Beyond Witnessing: Towards a Different Politics of the Sensible}

Throughout this paper I have argued that images of human suffering have critically shaped our understanding of tragedies that result from human rights abuses. I have tried to show how exposure to evidence of atrocities can affect an audience in different ways, and that the act of bearing witness should not be understood naively as a one-dimensional, individual experience, but rather as a political process that uses images and other media to sustain or advance specific political interests. I have also argued that, even if we become aware of how such processes affect us, the real challenge lies much deeper, namely in revealing how our subjectivity and agency are constituted through the effects of social forces that condition our capacity to perceive and understand. The main challenge that contemporary human rights advocates face, then, is posed not only by the controversial moral and political 
implications of the gruesome content of images, but also the ideological nature of processes woven within the images' use, reception and intentions that such exposure seeks to achieve. As I have suggested, public representations of atrocities may not only result in a fostering of humanitarian impulses; they may also numb public criticism and render agents unable to experience the world and other human beings in an engaged and caring way. The moral anxiety derived from witnessing distant suffering also brings us to another dilemma, namely, how we should understand this decay of human experience and whether there are ways to overcome the problem.

Given that patterns of exclusion feed on cross-cultural and historical contingencies, one can repeat Susan Sontag's concern that "our failure is one of imagination" (Sontag 2003: 8), and that the first step in overcoming the passivity of the viewing public may be to distance ourselves from the historical, cultural and economic structures that affect us. This echoes what Rancière writes in regard to an increasingly deceptive relation between ideology and social critique:

Forty years ago, critical science made us laugh at the imbeciles who took images for realities and let themselves be seduced by their hidden messages. In the interim the "imbeciles" have been educated in the art of recognizing the reality behind appearances and the messages concealed in images. And now, naturally enough, recycled critical science makes us smile at the imbeciles who still think such things, as concealed messages in images and a reality distinct from appearances exist. The machine can work in this way until the end of time, capitalizing on the impotence of the critique that unveils the impotence of the imbeciles (Rancière 2009: 48).

However much one might take issue with Rancière's claim, it is at least clear that the different ways in which social forces condition specific segments of an individual's experiences and subsequently his or her identity, leave the question unanswered as to how we may avoid this trap of what we might call perceptual predeterminism, and actually regain the capacity of critical reflection.

The aim of this final section, then, is not to repeat the criticism. I am not interested in prolonging the trial to which discourse on the political nature of images seems to be reduced. "If we want to have a fresh look at what images are, what they do and the effects they generate" (Rancière 2011: 95), disclosing what social powers frame as standards of intelligibility is just a first step toward emancipation. At stake here is not only a questioning of the privilege of the visual or an inevitable surrender to the inescapable nature of power, but also the hope of finding new paths to disturb the common ways in which perception is predisposed. In order to contest discursive visual norms that form a dominant system of experience and knowledge, an image needs to transcend, and at the same time remain part of, social experience. In other words, if aesthetic experience aims to have a critical effect on political conditions, it must disrupt the ways in which visual depictions of reality adhere to certain standards and functions. As Rancière writes, "The point is not to counter-pose reality to its appearances. It is to construct different realities, different forms of common sense." To contest mechanisms of domination and dehumanization, images, then, need to "help sketch new configurations of what can be seen, what can be said and what can be thought and, consequently, a new landscape of the possible. But they do so on condition that their 
meaning or effect is not anticipated" (Rancière 2011: 103). Only then can the "intolerable" in images really be taken as a serious medium of representation of human suffering, and not simply as a walk on a tightrope between victims and perpetrators viewed at a safe moral distance within the comfort of the western living room. To unsettle the framing of a visual culture that blurs the line between complicity and witnessing, as well as foster a "renewed confidence" in their political capacity, images must overcome the ideological veil of our existence by challenging what we are willing to know and how we react. They must prompt a refusal to ignore the political nature and implications of our numb spectatorship.

In the end, however plausible the previous insights may be, one can still argue that we are confronted with the difficult task of articulating the practical aspects of the obligations they entail. The initial worry of how to prompt audiences to adopt a more active, engaged political role when exposed to evidence of atrocities, brings us back to the starting question of this paper, namely, how to overcome increasing social indifference towards the suffering of others and acknowledge responsibilities that representations/existence of such conditions invoke. The difficulty here, then, is to recognize that, despite the moral disposition that such representations may produce, there still are no guarantees that such exposures would compel individuals to fulfill their obligations towards people in need. Although resolving the problem of motivation is not an explicit aim of this paper, I would like to conclude with a few brief suggestions of how representations of suffering may be made ethically effective means for better acknowledgment of individual and collective responsibility.

If Rancière and Butler's critique of standards of public representation poses the challenge of how to theorize the relationship between the political usage of images of atrocities and the formation of public sentiment about such events, their account of the situatedness of agency within interpretative frames only gestures towards possible sites of resistance. If we take such social and political structures as epistemological and ontological sites of subject formation, it remains unclear what direction a transformation of such agency should take and which normative implications should guide such processes. What we need here, then, is to take this moral residue that remains after we confront images of human agony and give the encounter a concrete political form. Acknowledging the tension between public representations of human suffering and the obligations that these representations place on spectators compounds the difficulties of formulating an ethics of responsibility. We must require not only an historically and institutionally specific reading of contemporary geopolitical conditions that result in human suffering, but also a detachment of depicted events from their concrete political contexts in which the problematic interpretations of these events take place. Despite all the admirable efforts to disclose mechanisms that prevent individual or collective acknowledgment of responsibilities aroused by evidence of human rights abuses, we still need a more careful vocabulary to distinguish between the conditions that result in human suffering, the conditions that frame its appearance, and the normative implications that such encounters demand from the viewing public. Without an account of how we may recognize the immediate humanitarian appeal that our privileged position as spectators carries within it, there will be no real grounds for political engagement after witnessing such intolerable instances of human deprivation. 
The pressing question, then, concerns what changes in our social setup are necessary in order to foster solidarity among people, even if we take into account a lack of commonality and complex geopolitical contexts that make some people more vulnerable to suffering than others. One way to address this issue is to conceive that the lives whose horrifying conditions we experience through visual representation of human suffering are already soliciting us both ethically and politically. Our visual apprehension of such imagery is an occasion in which implicit consent or dissent (engagement or apathy) to injustice and violence done to these people already formulates a social and ethical relation. If we take more seriously the complexity of this interconnection between spectator and victim we are compelled to extend our understanding of responsibility by indicating the privilege that spectators have, and what kind of obligations this privilege invokes. What most contemporary strategies of political activism seem to be lacking in the context of this relation between spectators and representations of atrocities is the insight that the moral appeal of human beings portrayed in their vulnerability is already part of the image. Put another way, moral implications towards the agonizing other are already part of the scene that we appropriate either as subjects who make such images or as those who only observe them. More importantly, there is a radical opposition between two kinds of perspectives that such images invoke. Both a spectator and photographer are in the privileged position with regard to the events that unfold in these images. This is not only because the events that these images depict are delivered over to public interpretation, but also because this asserted asymmetry is manifested in another, more radical, sense: both spectator and the image always "survive" indifference to victims exhibited through the latter.

I assume that a more nuanced account of this epistemic and ontological privilege is necessary if we want to engage concretely with the particular events that images exhibit. Taking into account that we are inevitably situated in a globalized world, we are called by the other to take a stand regarding events of human suffering. There is an ethical moment in this relationship between spectators and victims that we cannot completely deny, and its moral charge is grounded in the fact that we are always already involved in representational systems that place us in a hierarchical relationship to the persons affected by violent events. Therefore, it is not only necessary to interrogate the subjects whom are recruited as vessels of social powers and the "knowledge" that such powers want to convey: we must also show how the victims of these horrors are constituted by the same political subjectivity. If we want to affirm the political use of photography as a form of political activism that prompts public attention, one has to go beyond the cultural, psychological, and political aspects of its appearance and recuperate its moral implications. The outcome of this is profound, for if we attend to the asymmetries inherent in representations of suffering, we would be confronted with the need to revise some of our most basic presuppositions. These presuppositions concern not only our own political culture, but also in general what is meant when we conceive of humans or humanity. Thus, an ethics of responsibility begins with the acknowledgement that representations of others bear political and moral connotations, and that our reaction (or lack of reaction) towards the horrors depicted carries an inevitable risk that the choice we have made through our actions or disregard already constitutes a political stance that bears on moral evaluation. An image never stands alone, and once we come to terms with the inevitability of representation, both in terms of our 
own experience and the people mediated through them, then we must (and I suppose that "the must" here is the ethical moment) confront the following questions: how are we shaping others through those representations? Are we reinforcing the injustice done to them through our indifference and lack of commitment? What kinds of responsibilities are invoked? Do they go beyond our capacity to evaluate, articulate and engage with their sites of emergence?

For now, the problems persist. Maybe the lesson to be learned here is simply not to give up in the face of despair, to not remain blind to the lives that our inaction otherwise renders invisible. As Butler puts it, "( $\mathrm{t}$ )he precarity of life imposes an obligation upon us. We have to ask about the conditions under which it becomes possible to apprehend a life or set of lives as precarious, and those that make it less possible, or indeed impossible" (Butler 2010: 2). We must acknowledge, then, that human suffering is not just due to a chronic lack of reason and deviation from universal ethical norms. It is a complex social process within which subjects lose their ability to critically apprehend social maladies and identify their own complicity in sustaining the system. Ultimately, behind the façade of quasiemphatic responses and presentations in media, and alongside the interplay of apologetics and condemnations, there lies a core of numb blindness. This blindness deepens the gap between the capacity to see and to feel, and hence turns human witnessing into an imprecise anthropomorphic figure, a fictive boundary for the outlets created and consumed by the political public machine. Becoming aware of this relationship between representation, individual experience and social conditions will keep the embodied character of human deprivation from being ignored and/or made more palatable for collective reception. I am aware that this call to resist is neither new, nor without its challenges, but I also believe that it has the potential to call attention to subjects' positions in a web of historical and social conditions, and may contribute to their understanding of how interconnected and morally sensitive our world actually is.

\section{Notes}

${ }^{1}$ Mladjo Ivanonic is a $\mathrm{PhD}$ Candidate at the Department of Philosophy, Michigan State University. His doctoral thesis is called "Holding Hands with Death: How Does Representation of Human Suffering Constitute the Ethical Agent?". Ivanovic is the author of The Limits of Humanitarian Present (Review of Eyal Weizman (2011) The Least of All Possible Evils: Humanitarian Violence from Arendt to Gaza. New York: Verso) published in Radical Philosophy Review 2014: 17: 1.

${ }^{2}$ This is especially the case when the suffering one sees or reads about is happening to people living far away and with whom the audience is likely never to come into contact. How this political selectivism is grounded depends on the institutional capacity to mobilize public approval and convince their addressees to "rationally" endorse and apply these regulative norms in their everyday life.

${ }^{3}$ This notion of invisibility does not have to refer only to people who are not present as a content of visual representation, but also those individuals who are centerpiece of such images and still remain ignored and violated through the subsumption of a universal conceptual gaze of the commodified western public. 


\section{References}

Adorno, T. (2001). Can one live after Auschwitz? Philosophical reader. Ed. Rolf Tiedemann. Stanford: Stanford University Press.

Butler, J. (2007). Torture and the Ethics of Photography. Environment and Planning D: Society and Space 25: 951 - 966. http://dx.doi.org/10.1068/d2506jb

Butler, J. (2010). Frames of war. New York: Verso.

Butler, J. (2011). Politics of culture and the spirit of critique: dialogues. Ed. Gabriel Rockhill and Alfredo Gomez-Muller. New York: Columbia University Press.

Cohen, S. (2001). States of denial. Cambridge: Polity Press.

Dawes, J. (2007). That the world may know: bearing witness to atrocities. Cambridge: Harvard University Press. http://dx.doi.org/10.4159/9780674030275

Rancière, J. (2009). The future of the image. New York: Verso.

Rancière, J. (2011). The emancipated spectator. New York: Verso.

Sontag, S. (2003). Regarding the pain of others. New York: Picador.

Zelizer, B. (2000). Remembering to forget. Chicago: University of Chicago Press. 